\title{
Regensburger Nachwuchswissenschaftler ausgezeichnet
}

Für sein innovatives Forschungsprojekt zu regenerativen Therapien in der Zahnerhaltung ist Dr. Matthias Widbiller, Assistenzarzt der Poliklinik für Zahnerhaltung und Parodontologie des Universitätsklinikums Regensburg (UKR), mit zwei renommierten Forschungspreisen ausgezeichnet worden.

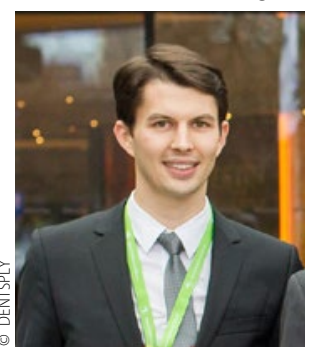

Mit diesem Themenfeld befasst sich Widbiller in der Forschungsgruppe um Professor Dr. Kerstin Galler, Oberärztin der Poliklinik für Zahnerhaltung und Parodontologie des UKR. Die Ergebnisse seines aktuellen Forschungsprojekts könnten Zahnärzten völlig neue Therapiemöglichkeiten eröffnen. Beispielsweise könnte bei jungen Pati- enten mit abgestorbenem Zahnmark die Zahnwurzel wieder zum Wachstum angeregt werden.

Für seine Forschungsarbeit wurde Widbiller im November 2015 mit dem mit 20.000 Euro dotierten Forschungsförderpreis der Deutschen Gesellschaft für Restaurative und Regenerative Zahnerhaltung (DGR2Z) sowie mit dem DENTSPLY Förderpreis 2015 ausgezeichnet, der beim Deutschen Zahnärztetag in Zusammenarbeit mit der Bundeszahnärztekammer und der Deutschen Gesellschaft für Zahn-, Mund- und Kieferheilkunde verliehen wird.

Den zweiten DENTSPLY Förderpreis in dieser Kategorie erhielt Tanja Basan, (Universität Rostock). Susanne Faber (Doktorandin an der Universität Witten/Herdecke) wurde in der Kategorie Klinische Verfahren und Behandlungsmethoden für ihre Arbeit über Zahnarzt-Angst mit dem 2. Preis ausgezeichnet.

Schnelle Streifen für YouTube, Twitter \& Co.

\section{ProDente kürt Gewinner des Kurzfilmwettbewerbs}

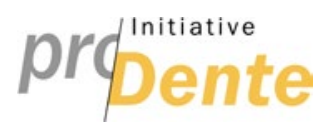

Die Initiative proDente hat die drei Gewinner des Kurzfilmwettbewerbs „Zähne gut - alles gut“ bei der Preisverleihung in Köln gekürt. Studenten, Schüler und freie Filmkünstler waren dazu aufgerufen, Kurzfilme rund um ,schöne und gesunde Zähne“ zu drehen. Der Kurzfilmwettbewerb lief über YouTube, Facebook und Twitter und sollte Menschen in den sozialen Medien auf das Thema Zähne aufmerksam machen. Aus 50 Einsendungen ermittelte die Jury drei Gewinner, die sich den mit 6.000 Euro dotierten Preis teilen. 140.000 Zuschauer sahen sich den Film der Erstplatzierten Antonia Kaehn „Zähne sind nicht gern allein“ an, der zum Publikumsliebling des Wettbewerbs avancierte. Die Jury war von der einfallsreichen Idee und der emotionalen Umsetzung be- geistert. Platz zwei ging an Philipp Letz für den Film „Deine Story“. Der Film sei „toll erzählt, zweideutig, ironisch und emotional“ urteilte die Jury und vor allem „sehr unterhaltsam“. Lars Schnell landete mit „Waltz of Gore“ auf Platz drei - ein Film, in dem die Angst im Mittelpunkt steht. Ziel des Filmwettbewerbs ist es, junge Menschen langfristig für das Thema Zähne zu begeistern. „Das Preisgeld soll die jungen Talente dabei unterstützen, weiterhin so kompetente und spannende Projekte zu realisieren“, sagte Kropp bei der Preisverleihung in Köln. Zu den Juroren 2015 gehörten die Kölner Schauspielerin Julia Schmitt, Ben Redelings, Autor, Filmemacher und Fußball-Komiker aus Bochum, sowie proDente-Geschäftsführer Kropp.

\section{Nachruf}

\section{Ehemaliger apoBank-Chef Schlenkenbrock in hohem Alter verstorben}

Der Ehrenvorsitzende und ehemalige Vorsitzende des Vorstands der Deutschen Apotheker- und Ärztebank (apoBank), DiplomVolkswirt Walter Schlenkenbrock, ist im Alter von 90 Jahren verstorben. Schlenkenbrock war 33 Jahre für die apoBank tätig und stand von 1970 bis 1990 an deren Spitze. Die Ära Schlenkenbrock steht unter anderem für den massiven Ausbau des Fi- lialnetzes, die Intensivierung der betriebswirtschaftlichen Beratung sowie den Aufbau und die Festigung der Verbindungen zu den Standesorganisationen. Schlenkenbrock war Inhaber des Ehrenzeichens der deutschen Ärzteschaft, Träger der Ehrennadel der Deutschen Apotheker sowie der Ehrennadel der deutschen Zahnärzteschaft in Gold.

red

\section{Einstimmiges Votum}

\section{Martin Litsch ist neuer AOK-Chef}

Der AOK-Bundesverband hat einen neuen Chef: Der Aufsichtsrat wählte Ende November Martin Litsch, der zuvor die AOK Nordwest führte, einstimmig zum Vorstandsvorsitzenden. Litsch hatte den AOK-Bundesverband bereits seit dem vergangenen Sommer als Interimsvorstand geleitet. Der neue AOK-Chef trat sein Amt zum Jahreswechsel an. Nach einem Machtkampf über die Zukunft des Verbands hatten seine Vorgänger Jürgen Graalmann und Uwe Deh im Juli 2015 zeitgleich ihre Posten niedergelegt. 